\title{
Global $\Lambda$ polarization in moderately relativistic nuclear collisions
}

\author{
Yu. B. Ivanov $1,2,3$, 田 \\ ${ }^{1}$ Bogoliubov Laboratory for Theoretical Physics, Joint Institute for Nuclear Research, Dubna 141980, Russia \\ ${ }^{2}$ National Research Nuclear University "MEPhI", Moscow 115409, Russia \\ ${ }^{3}$ National Research Center "Kurchatov Institute", Moscow 123182, Russia
}

\begin{abstract}
Predictions for the global polarization of $\Lambda$ hyperons in $\mathrm{Au}+\mathrm{Au}$ collisions at moderately relativistic collision energies, $2.4 \leq \sqrt{s_{N N}} \leq 11 \mathrm{GeV}$, are made. These are based on the thermodynamic approach to the global polarization incorporated into the model of the three-fluid dynamics. Centrality dependence of the polarization is studied. It is predicted that the polarization reaches a maximum or a plateau (depending on the equation of state and centrality) at $\sqrt{s_{N N}} \approx 3 \mathrm{GeV}$. It is found that the global polarization increases with increasing width of the rapidity window around the midrapidity.
\end{abstract}

PACS numbers: $25.75 .-\mathrm{q}, 25.75 . \mathrm{Nq}, 24.10 . \mathrm{Nz}$

Keywords: relativistic heavy-ion collisions, hydrodynamics, polarization

Experimental discovery of global polarization of $\Lambda$ and $\bar{\Lambda}$ hyperons in the STAR experiment [1, 2] gave us evidence of existence of a new class of collective phenomena in heavy-ion collisions. The global polarization of hyperons is well described within the thermodynamic approach based on hadronic degrees of freedom 3 5. This was demonstrated by implementation of this approach into various hydrodynamical 6-11 and transport [1217. models of heavy-ion collisions. This thermodynamic approach is not without its problems, see recent review in Ref. [18. One of them, concerning the the global polarization, is that it does not explain large difference between $\Lambda$ and $\bar{\Lambda}$ polarization at $7.7 \mathrm{GeV}$. Various explanations of this $\Lambda-\bar{\Lambda}$ splitting were put forward [17, 19,23]. However, it is not yet quite clear how severe this problem of splitting is because the $\bar{\Lambda}$ polarization at $7.7 \mathrm{GeV}$ is measured with poor accuracy.

At present, experiments at lower collision energies than those of the Beam Energy Scan (BES) program at the Relativistic Heavy Ion Collider (RHIC) are in progress: STAR fixed target (FXT) program [24] at RHIC and HADES [25] at GSI Helmholtzzentrum für Schwerionenforschung. Also new facilities for heavy-ion collisions are under construction, which are designed for collider regime, Nuclotron-based Ion Collider fAcility (NICA) in Dubna [26, and for fixed-target experiments, the Baryonic Matter at Nuclotron $(\mathrm{BM} @ N)^{1}$ in Dubna [27, 28, the Facility for Antiproton and Ion Research (FAIR) in Darmstadt 29, and High Intensity heavy-ion Accelerator Facility (HIAF) in Huizhou, China [30. The energy ranges for the $\mathrm{Au}$ beams are $\sqrt{s_{N N}}=3-7.2 \mathrm{GeV}$ for STAR-FXT, $\sqrt{s_{N N}}=2.3-2.6 \mathrm{GeV}$ for HADES, $\sqrt{s_{N N}}$ $=2.3-3.5 \mathrm{GeV}$ for $\mathrm{BM} @ \mathrm{~N}, \sqrt{s_{N N}}=2.3-4 \mathrm{GeV}$ for $\mathrm{HIAF}, \sqrt{s_{N N}}=2.7-4.9 \mathrm{GeV}$ for FAIR, and $\sqrt{s_{N N}}=$

*e-mail: yivanov@theor.jinr.ru

1 In fact, the BM@N is already in operation, but the planed beams of really heavy ions with high luminosity will be achieved only in future.
$4-11 \mathrm{GeV}$ for NICA. Measurements of the global $\Lambda$ polarization are already in progress at the STAR-FXT and HADES [43].

The NICA and BES-RHIC energy ranges overlap. Therefore, all above mentioned calculations of the global polarization [6-17, 22, 23] involved the high-energy end of the NICA range. Even some works dedicated to the polarization at NICA and FAIR energies [7, 16] in fact considered the upper end of this range that overlaps with BES-RHIC energies. Predictions of the global polarization in the actual NICA range were done in Refs. 911, 22, 23. The first estimates at even lower energies but in terms of vorticity were recently reported in Ref. 31. All the estimates agree with that the global polarization (vorticity 31) rises with the collision energy decrease. Ref. 31 predicts that the maximum vorticity is reached at $\sqrt{s_{N N}} \approx 3 \mathrm{GeV}$ and then it decreases with decreasing collision energy.

In this paper, predictions for the global polarization of $\Lambda$ hyperons at HADES-NICA energies are made, based on the thermodynamic approach [3 5]. Simulations are performed within the model of the three-fluid dynamics (3FD) 32. The 3FD model takes into account nonequilibrium at the early stage of nuclear collisions. This nonequilibrium stage is modeled by means of two counterstreaming baryon-rich fluids. Newly produced particles, dominantly populating the midrapidity region, are attributed to a fireball fluid. These fluids are governed by conventional hydrodynamic equations coupled by friction terms in the right-hand sides of the Euler equations. Calculations are done with three different equations of state (EoS's): a purely hadronic EoS [33] and two versions of the EoS with the deconfinement transition [34, i.e. a first-order phase transition (1PT) and a crossover one. The physical input of the present 3FD calculations is described in Ref. 35.

In Fig. 1 the global polarization of $\Lambda$ hyperons in $\mathrm{Au}+\mathrm{Au}$ collisions at different centralities, i.e. impact parameters $b=2,4,6$ and $8 \mathrm{fm}$, is presented in the collisionenergy range extended to the low energies. The STAR data [1] are also displayed to connect the low-energy pre- 
dictions with the data available at BES-RHIC. The impact parameter $b=8 \mathrm{fm}$ roughly complies with the STAR centrality selection of $20-50 \%$ [1]. Glauber simulations of Ref. 36] were used to relate the experimental centrality and the mean impact parameter. In the 3FD model, the colliding nuclei have a shape of sharp spheres without the Woods-Saxon diffuse edge. This fact, implemented in the Glauber simulations, results in a shift of the impact parameter to a value lower by $\approx 1.5 \mathrm{fm}$ as compared with the results of Ref. 36. The nuclear overlap calculator [37. was used for this estimate.

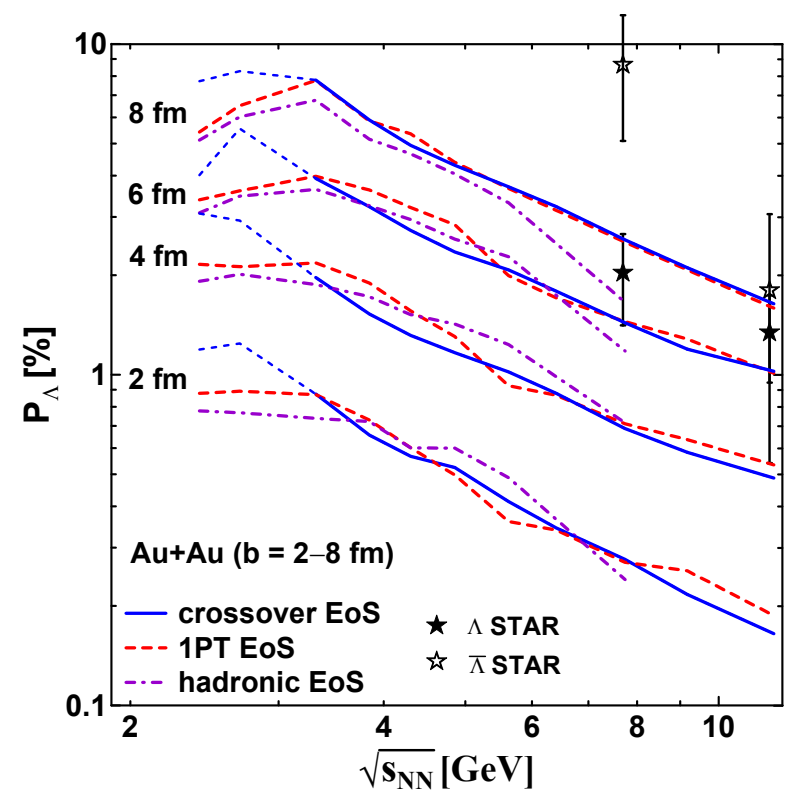

FIG. 1: (Color online) Global polarization of $\Lambda$ hyperons in $\mathrm{Au}+\mathrm{Au}$ collisions at impact parameters $b=2,4,6,8 \mathrm{fm}$ as function of collision energy $\sqrt{s_{N N}}$ calculated with different EoS's within the framework of the thermodynamical approach. Short-dashed lines for the crossover EoS below $3 \mathrm{GeV}$ indicate the unstable numerics. STAR data on global $\Lambda$ and $\bar{\Lambda}$ polarization 1] are also displayed.

The polarization in Fig. 1 is calculated precisely in the same way as it is described in Ref. [11. It is related to the midrapidity region $\left|y_{h}\right| \lesssim 0.5$, where rapidity range is calculated based on hydrodinamical velocities. This constraint roughly simulates the STAR acceptance in terms of pseudorapidity $|\eta|<1$. Of course, the acceptance in different experiments may be different, the STAR acceptance is implemented just for definiteness. The above constraint means that at the freeze-out instant the polarization is averaged over the central region of colliding nuclei confined by the condition $\left|y_{h}\right| \lesssim 0.5$. As this condition is related to a certain choice of spatial width of this central region, its rapidity width $\Delta y_{h}$ slightly depends on the collision energy, centrality $(b)$ and EoS, such that $\Delta y_{h} / 2 \approx 0.5$. $\Delta y_{h} / 2$ is not exactly equal to 0.5 , e.g., it is 0.45 at $\sqrt{s_{N N}}=2.42 \mathrm{GeV}$. For details of this calculation, please, refer to Ref. 11]. The failure of the hadronic EoS at $\sqrt{s_{N N}}>8 \mathrm{GeV}$ is discussed in Ref. [11] in detail. To avoid this discussion that is irrelevant to the low energies considered here, the hadronic-EoS results are displayed only below the energy of $8 \mathrm{GeV}$.

The global polarization predicted by the crossover and 1PT EoS's is very similar at $\sqrt{s_{N N}}>3 \mathrm{GeV}$, while at $\sqrt{s_{N N}}<3 \mathrm{GeV}$ the predictions differ. Predictions of the hadronic EoS slightly differ from two above even at $3<\sqrt{s_{N N}}<7 \mathrm{GeV}$. The reason is that the hadronic sectors of the crossover, 1PT and hadronic EoS's are similar but not identical. Therefore, the difference between results of different EoS's characterizes uncertainty of the present prediction. The numerics of simulations with the crossover EoS becomes unstable at $\sqrt{s_{N N}}<3 \mathrm{GeV}$. Apparenly, this is a consequence of somewhat poor quality of tabulation of this EoS at low densities. This could be a reason of the large difference of the crossover results from other predictions. Nevertheless, these low-energy crossover results are displayed in Fig. 1 by short-dashed lines.

As seen from Fig. 1, the global polarization increases with the collision energy decrease. Depending on the EoS, it reaches values of $6-8 \%$ at $\sqrt{s_{N N}}=3.3 \mathrm{GeV}$ at $b=8 \mathrm{fm}$. At $\sqrt{s_{N N}} \approx 3 \mathrm{GeV}$ this increase slows down or even a maximum is reached, again depending on the EoS and centrality. This is in agreement with findings in Ref. [31. Note the the decrease of the global polarization with the energy rise was predicted in Ref. 38] long before the first experimental results. That prediction was based on the chiral vortical effect.

The appearance of a maximum in the global polarization is very natural. On the one hand, the polarization tends to zero when $\sqrt{s_{N N}}$ approaches to $2 m_{N}$ (two nucleon masses) because the total angular momentum of the colliding system becomes close to zero. on the other hand, the polarization rises with increase of the collision energy and hence the total angular momentum. For the total polarization, i.e. that averaged over the whole range of rapidities, this is a monotonous increase 9 , 10. However, the global polarization is measured in the midrapidity region. As found both experimentally and theoretically the the global polarization in the BES-RHIC region decreases with the energy rise. This happens because the vorticity and hence the polarization migrates to backward-forward rapidities $9,[10,39-42$. This migration produces the maximum in the global polarization. The position and the height of this maximum depends on the width of the midrapidity window and on the dynamics of the vorticity migration to the peripheral rapidities.

Preliminary result by the HADES Collaboration [43] was measured at $\sqrt{s_{N N}}=2.4 \mathrm{GeV}$ and the centrality of $10-40 \%$. It best of all corresponds to $b=6 \mathrm{fm}$ among the calculated impact parameters. Although this result is still not quite reliably extracted against the background, it does not contradict the 3FD prediction at $b=6 \mathrm{fm}$.

The effect of the rapidity acceptance is demonstrated in Fig. 2, where results at $b=8 \mathrm{fm}$ are presented for rapidity windows: $\left|y_{h}\right| \lesssim 0.5$ (displayed lines), $\left|y_{h}\right| \lesssim 0.6$ (upper borders of the correspondingly colored bands) and 
$\left|y_{h}\right| \lesssim 0.35$ (lower borders of these bands). The global polarization is presented in a wider energy range, 2.4 $\geq \sqrt{s_{N N}} \geq 39 \mathrm{GeV}$, to see the effect at various collision energies. At higher collision energies, the effect is small because even the wider rapidity range, $\left|y_{h}\right| \lesssim 0.6$, amounts a small fraction of the total one. The effect becomes stronger with the energy decrease, reaching its maximum at $\approx 3 \mathrm{GeV}$. Surprisingly, the effect again becomes small at $2.4 \mathrm{GeV}$. Similar results for other centralities, $b=6$ and $4 \mathrm{fm}$, are presented in Figs. 3 and 4. respectively. The STAR data are presented there as benchmarks for comparing the scale at different centralities. The crossover bands are displayed only above 3.3 $\mathrm{GeV}$, because of unstable numerics at lower energies.

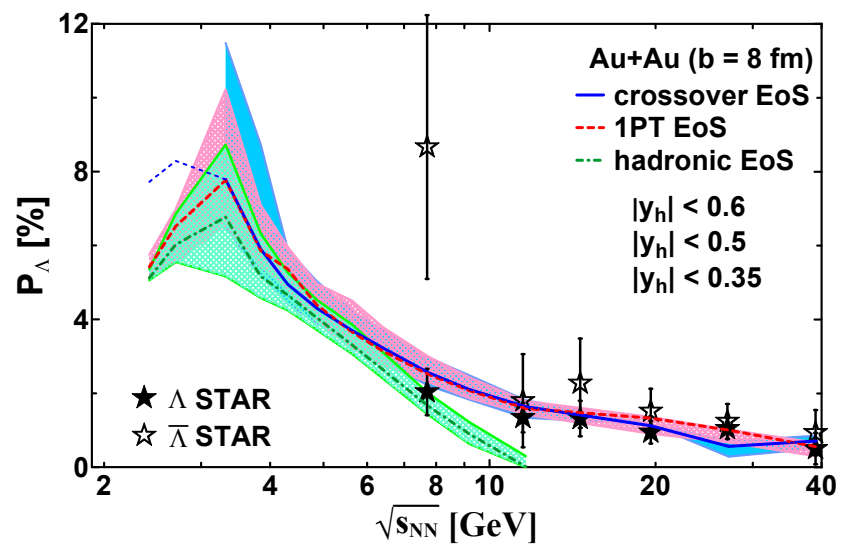

FIG. 2: (Color online) Global polarization of $\Lambda$ hyperons in $\mathrm{Au}+\mathrm{Au}$ collisions at $b=8 \mathrm{fm}$ as function of collision energy $\sqrt{s_{N N}}$ calculated with different EoS's. The lines correspond to the midrapidity region constrained by the $\left|y_{h}\right| \lesssim 0.5$ condition. The upper borders of the correspondingly colored bands around these lines correspond to the constraint $\left|y_{h}\right| \lesssim 0.6$, while the lower borders - to $\left|y_{h}\right| \lesssim 0.35$. STAR data on global $\Lambda$ and $\bar{\Lambda}$ polarization [1] are also displayed.

In the present calculation the same freeze-out for all species is used. As a result the $\bar{\Lambda}$ polarization turned out to be very close to the $\Lambda$ one, therefore it is not presented here. Earlier freeze-out of $\bar{\Lambda}$ 's partially solves the problem of the $\Lambda-\bar{\Lambda}$ splitting [17, but not at the lowest BES-RHIC energy of $7.7 \mathrm{GeV}$.

Feed-down contribution to the $\Lambda$ polarization due to decays of higher mass hyperons is not taken into account in the present estimate. This feed-down can reduce the polarization by about $10-15 \%$, as demonstrated in Refs. [5, 6, 18, 44, 45, albeit at higher collision energies. This feed-down effect is definitely of minor importance at low collision energies because of low abundances of higher mass hyperons.

An alternative approach to the global $\Lambda$ polarization is based on the Axial Vortical Effect (AVE) [46 49, which is in fact identical to the Chiral Vortical Effect (CVE). It reasonably good describes the data on the global polarization 22, 23] and naturally explains the above mentioned $\Lambda-\bar{\Lambda}$ splitting [22, 23. However, in the energy

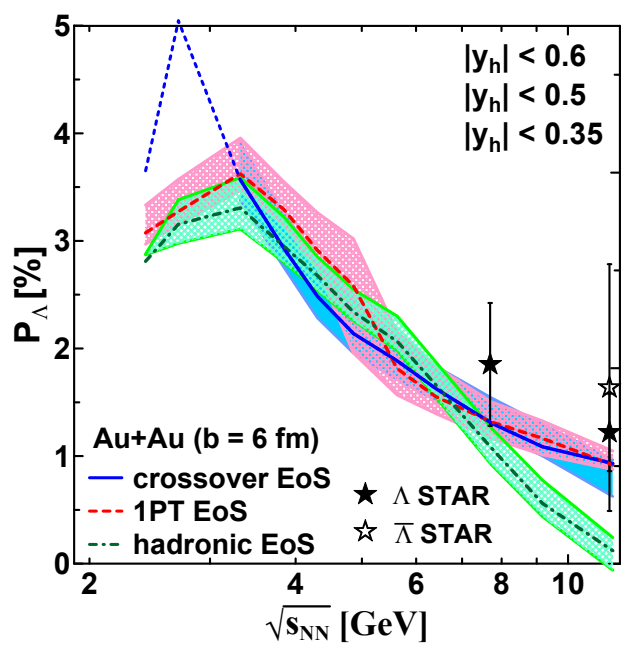

FIG. 3: (Color online) The same as in Fig. 2 but for $b=6$ fm.

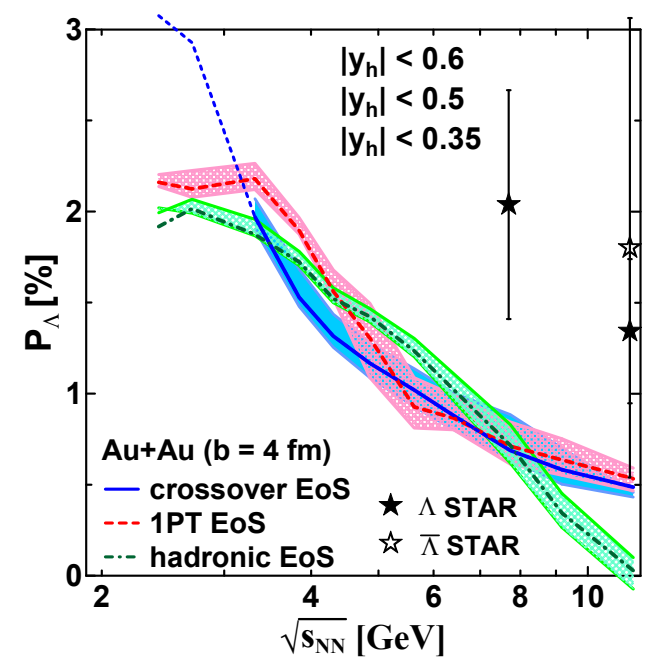

FIG. 4: (Color online) The same as in Fig. 2 but for $b=4$ fm.

range below the NICA region it is hardly applicable because the chiral symmetry, i.e. the driving force of the AVE, is spontaneously broken.

To summarize, based on the 3FD model, predictions for the global $\Lambda$ polarization in $\mathrm{Au}+\mathrm{Au}$ collisions in current and upcoming experiments at moderately relativistic energies, $2.4 \leq \sqrt{s_{N N}} \leq 11 \mathrm{GeV}$, are made. It is predicted that the polarization reaches a maximum or a plateau (depending on the EoS and centrality) at $\sqrt{s_{N N}} \approx 3 \mathrm{GeV}$. It is found that the global polarization increases when the width of the rapidity window around the midrapidity rises. The preliminary result of HADES Collaboration [43, although not yet quite reliably extracted against the background, does not contradict the results of the present calculation. Of course, not all related aspects were considered in this short paper, e.g. a more detailed study of the rapidity dependence of the 
global polarization is required, applicability of the thermodynamic approach to nuclear collisions at low energies (the collision dynamics becomes less equilibrium with the collision energy decrease) should be checked, etc. These topics will be considered elsewhere.

Helpful discussions with F. Becattini, O.V. Teryaev, V.D. Toneev, and D.N. Voskresensky are gratefully acknowledged. This work was carried out using computing resources of the federal collective usage cen- ter "Complex for simulation and data processing for mega-science facilities" at NRC "Kurchatov Institute", http://ckp.nrcki.ru/. This work was partially supported by the Russian Foundation for Basic Research, Grants No. 18-02-40084 and No. 18-02-40085, and by the Ministry of Education and Science of the Russian Federation within the Academic Excellence Project of the NRNU MEPhI under contract No. 02.A03.21.0005.
[1] L. Adamczyk et al. [STAR Collaboration], Nature 548, 62 (2017) arXiv:1701.06657 [nucl-ex]].

[2] J. Adam et al. [STAR Collaboration], Phys. Rev. C 98, 014910 (2018) arXiv:1805.04400 [nucl-ex]].

[3] F. Becattini, V. Chandra, L. Del Zanna and E. Grossi, Annals Phys. 338, 32 (2013) arXiv:1303.3431 [nucl-th]].

[4] R. h. Fang, L. g. Pang, Q. Wang and X. n. Wang, Phys. Rev. C 94, no. 2, 024904 (2016) arXiv:1604.04036 [nuclth]].

[5] F. Becattini, I. Karpenko, M.A. Lisa, I. Upsal and S.A. Voloshin, Phys. Rev. C 95, no. 5, 054902 (2017) arXiv:1610.02506 [nucl-th]].

[6] I. Karpenko and F. Becattini, Eur. Phys. J. C 77, no. 4, 213 (2017) arXiv:1610.04717 [nucl-th]].

[7] Y. L. Xie, M. Bleicher, H. Stocker, D. J. Wang and L. P. Csernai, Phys. Rev. C 94, no. 5, 054907 (2016) arXiv:1610.08678 [nucl-th]].

[8] Y. Xie, D. Wang and L. P. Csernai, Phys. Rev. C 95, no. 3, 031901 (2017) arXiv:1703.03770 [nucl-th]].

[9] Y. B. Ivanov, V. D. Toneev and A. A. Soldatov, Phys. Rev. C 100, no. 1, 014908 (2019) arXiv:1903.05455 [nucl-th]].

[10] Y. B. Ivanov, V. D. Toneev and A. A. Soldatov, Phys. Atom. Nucl. 83, no. 2, 179 (2020). arXiv:1910.01332 [nucl-th]].

[11] Y. B. Ivanov and A. A. Soldatov, Phys. Rev. C 102, no.2, 024916 (2020) arXiv:2004.05166 [nucl-th]].

[12] H. Li, L. G. Pang, Q. Wang and X. L. Xia, Phys. Rev. C 96, no. 5, 054908 (2017) arXiv:1704.01507 [nucl-th]].

[13] Y. Sun and C. M. Ko, Phys. Rev. C 96, no. 2, 024906 (2017) arXiv:1706.09467 [nucl-th]].

[14] D. X. Wei, W. T. Deng and X. G. Huang, Phys. Rev. C 99, no. 1, 014905 (2019) arXiv:1810.00151 [nucl-th]].

[15] S. Shi, K. Li and J. Liao, Phys. Lett. B 788, 409 (2019) arXiv:1712.00878 [nucl-th]].

[16] E. E. Kolomeitsev, V. D. Toneev and V. Voronyuk, Phys. Rev. C 97, no. 6, 064902 (2018) arXiv:1801.07610 [nuclth]].

[17] O. Vitiuk, L. V. Bravina and E. E. Zabrodin, Phys. Lett. B 803, 135298 (2020) arXiv:1910.06292 [hep-ph]].

[18] F. Becattini and M. Lisa, Ann. Rev. Nucl. Part. Sci. 70, 395-423 (2020) arXiv:2003.03640 [nucl-ex]].

[19] L.P. Csernai, J.I. Kapusta and T. Welle, Phys. Rev. C 99, no.2, 021901(R) (2019) arXiv:1807.11521 [nucl-th]].

[20] Y. Xie, G. Chen and L. P. Csernai, Eur. Phys. J. C 81, no.1, 12 (2021) arXiv:1912.00209 [hep-ph]].

[21] A. Ayala, M. A. Ayala Torres, E. Cuautle, I. Domínguez, M. A. Fontaine Sanchez, I. Maldonado, E. MorenoBarbosa, P. A. Nieto-Marín, M. Rodríguez-Cahuantzi and J. Salinas, et al. Phys. Lett. B 810, 135818 (2020) arXiv:2003.13757 [hep-ph]].

[22] M. Baznat, K. Gudima, A. Sorin and O. Teryaev, Phys. Rev. C 97, no. 4, 041902(R) (2018) arXiv:1701.00923 [nucl-th]].

[23] Y. B. Ivanov, Phys. Rev. C 102, no.4, 044904 (2020) arXiv:2006.14328 [nucl-th]].

[24] K. Meehan [STAR], Nucl. Phys. A 967, 808-811 (2017) arXiv:1704.06342 [nucl-ex]].

[25] G. Agakishiev et al. [HADES], Eur. Phys. J. A 41, 243277 (2009) arXiv:0902.3478 [nucl-ex]].

[26] V. D. Kekelidze, V. A. Matveev, I. N. Meshkov, A. S. Sorin and G. V. Trubnikov, Phys. Part. Nucl. 48, no. 5, 727 (2017).

[27] M. Kapishin [BM@N], Nucl. Phys. A 982, 967-970 (2019)

[28] BM@N Conceptual Design Report: http://nica.jinr.ru/files/BM@N/BMN_CDR.pdf

[29] T. Ablyazimov et al. [CBM], Eur. Phys. J. A 53, no.3, 60 (2017) arXiv:1607.01487 [nucl-ex]].

[30] L. Lü, H. Yi, Z. Xiao, M. Shao, S. Zhang, G. Xiao and N. Xu, Sci. China Phys. Mech. Astron. 60, no.1, 012021 (2017) doi:10.1007/s11433-016-0342-x

[31] X. G. Deng, X. G. Huang, Y. G. Ma and S. Zhang, Phys. Rev. C 101, no.6, 064908 (2020) arXiv:2001.01371 [nuclth]].

[32] Yu. B. Ivanov, V. N. Russkikh, and V.D. Toneev, Phys. Rev. C 73, 044904 (2006) nucl-th/0503088.

[33] I. N. Mishustin, V. N. Russkikh and L. M. Satarov, Sov. J. Nucl. Phys. 54, 260-314 (1991)

[34] A. S. Khvorostukhin, V. V. Skokov, K. Redlich, and V. D. Toneev, Eur. Phys. J. C48, 531 (2006) nucl-th/0605069.

[35] Yu. B. Ivanov, Phys. Rev. C 87, 064904 (2013) arXiv:1302.5766 [nucl-th]].

[36] B. I. Abelev et al. [STAR Collaboration], Phys. Rev. C 79, 034909 (2009) arXiv:0808.2041 [nucl-ex]].

[37] http://web-docs.gsi.de/ misko/overlap/interface.html

[38] O. Rogachevsky, A.S. Sorin and O.V. Teryaev, Phys. Rev. C 82, 054910 (2010) arXiv:1006.1331 [hep-ph]].

[39] Y. B. Ivanov and A. A. Soldatov, Phys. Rev. C 97, no.4, 044915 (2018) arXiv:1803.01525 [nucl-th]].

[40] Y. B. Ivanov and A. A. Soldatov, Phys. Rev. C 95, no.5, 054915 (2017) arXiv:1701.01319 [nucl-th]].

[41] M. I. Baznat, K. K. Gudima, A. S. Sorin and O. V. Teryaev, Phys. Rev. C 93, no. 3, 031902(R) (2016) arXiv:1507.04652 [nucl-th]].

[42] M. Baznat, K. Gudima, A. Sorin and O. Teryaev, Phys. Rev. C 88, no. 6, 061901(R) (2013) arXiv:1301.7003 [nucl-th]].

[43] F. J. Kornas [HADES], Springer Proc. Phys. 250, 435- 
439 (2020).

[44] F. Becattini, G. Cao and E. Speranza, Eur. Phys. J. C 79, no.9, 741 (2019) arXiv:1905.03123 [nucl-th]].

[45] X. L. Xia, H. Li, X. G. Huang and H. Z. Huang, Phys. Rev. C 100, no.1, 014913 (2019) arXiv:1905.03120 [nuclth]].

[46] A. Vilenkin, Phys. Rev. D 21, 2260 (1980).

[47] D.T. Son and A. R. Zhitnitsky, Phys. Rev. D 70, 074018
(2004) arXiv:hep-ph/0405216 [hep-ph]].

[48] J. H. Gao, Z. T. Liang, S. Pu, Q. Wang and X. N. Wang, Phys. Rev. Lett. 109, 232301 (2012) arXiv:1203.0725 [hep-ph]].

[49] A. Sorin and O. Teryaev, Phys. Rev. C 95, no. 1, 011902(R) (2017) arXiv:1606.08398 [nucl-th]]. 